\title{
Regularity analysis for optimizing urban transit network design
}

\author{
Niels van Oort $\cdot$ Rob van Nes
}

Published online: 7 August 2009

(C) The Author(s) 2009. This article is published with open access at Springerlink.com

\begin{abstract}
Transit network planners often propose network structures that either assume a certain level of regularity or are even especially focused on improving service reliability, such as networks in which parts of lines share a common route or the introduction of short-turn services. The key idea is that travelers on that route will have a more frequent transit service. The impact of such network designs on service regularity is rarely analyzed in a quantitative way. This paper presents a tool that can be used to assess the impact of network changes on the regularity on a transit route and on the level of transit demand. The tool can use actual data on the punctuality of the transit system. The application of such a tool is illustrated in two ways. A case study on introducing coordinated services shows that the use of such a tool leads to more realistic estimates than the traditional approach. Second, a set of graphs is developed which can be used for a quick scan when considering network changes. These graphs can be used to assess the effect of coordinating the schedules and of improving the punctuality.
\end{abstract}

\section{Introduction}

Service regularity is a key service quality characteristic of transit systems, especially for high frequency transit services such as in urban areas. Travelers arrive at the stop randomly, and if the services are performed regularly, the waiting time will be equal

\footnotetext{
N. van Oort $(\varangle)$

Department of Research and Development, HTM Urban Transport Company, P.O. Box 28503, 2502 KM The Hague, The Netherlands e-mail: N.van.Oort@HTM.net

R. van Nes

Faculty of Civil Engineering and Geosciences, Transport \& Planning, Delft University of Technology, P.O. Box 5048, 2600 GA Delft, The Netherlands

e-mail: R.vanNes@tudelft.nl
} 
to half the headway. In case of more lines sharing tracks, coordination is necessary to achieve minimal waiting times. In urban transit networks, however, it is nearly impossible to have $100 \%$ regular services. Vehicles use the same infrastructure as private cars, and therefore suffer from all kinds of delays such as congestion, traffic lights, et cetera. Furthermore, drivers have different characteristics, while the process of boarding and alighting will vary due to variety in characteristics and numbers of passengers per stop. The net result is that travelers perceive longer waiting times than expected, while more vehicles are needed to provide adequate capacity. Assuring regular transit services is thus an important task for transit service companies.

Many studies focus on operational measures for improving service regularity, first of all by monitoring the actual service performance and by informing drivers of their actual situation (Muller and Furth 2000a, 2000b; Osuna and Newell 1972; Chowdhury and Chien 2001; Fu and Liu 2003). Others deal with timetable design and service regularity, analyzing issues such as determining realistic trip times and including buffers for controlling the service quality (Carey 1998; Israeli and Ceder 1996). The combination of service regularity and transit network planning in case of urban transport is rarely analyzed.

In practice however, transit network designers also propose network structures that either assume a certain level of service regularity or are even especially focused on improving the service reliability. A typical example of such a network structure is the case where (parts of) two or more transit lines are running parallel on the same route. The basic idea is that travelers on that route will have a more frequent transport service. Another strategic planning measure is the introduction of additional transit services for the more heavily used parts of a transit line (short-turn services). Although these network designs clearly have a relationship with service regularity, the impact on service regularity is often only analyzed in a qualitative way. Since the key question in transit network design is what is achieved in terms of demand versus the related costs, it is obvious that a quantitative analysis would be more appropriate. This paper proposes a tool that a transit network planner can use to assess the impact of network changes on the service regularity.

The paper is structured as follows: Section 2 provides a discussion on regularity and punctuality and their impact on travelers and the level of demand. Section 3 describes the framework of the tool for analyzing the service regularity when designing an urban transit network. Sections 4 and 5 illustrate how the tool can be used when considering specific network changes and for quick scans, respectively. Finally, Sect. 6 summarizes the conclusions.

\section{Regularity, punctuality and travel demand}

Regularity and punctuality are two closely related concepts. This section defines both service quality characteristics and discusses the impact of irregular services on travel time and on travel behavior.

\subsection{Definitions}

The regularity of a transport service is determined by the variation in its headways. This is caused by a variation in trip times and boarding and alighting times, for in- 
stance due to weather conditions, traffic lights, other traffic, congestion, driver behavior, and number and characteristics of travelers boarding and alighting (Barnett 1974). One way to describe the regularity of a single transport line is by using the PRDM (Percentage regularity deviation mean) (Hakkesteegt and Muller 1981).

$$
\operatorname{PRDM}_{j}=\frac{\sum_{i}\left|\frac{H_{i, j}-H_{i, j}^{\prime}}{H_{i, j}}\right|}{n_{j}}
$$

where:

$$
\begin{aligned}
\operatorname{PRDM}_{j} & =\text { relative regularity for stop } j \\
H_{i, j} & =\text { scheduled headway for vehicle } i \text { at stop } j \\
H_{i, j}^{\prime} & =\text { actual headway for vehicle } i \text { at stop } j \\
n_{j} & =\text { number of vehicles serving stop } j
\end{aligned}
$$

The PRDM is defined such that if its value is $0 \%$ regularity is perfect, while a value of $100 \%$ implies bunched arrivals. This indicator is commonly used in The Netherlands and is included in the TRITAPT-monitoring system (TU Delft 1997-2002). In case of more lines on a track, offering a combined, uneven headway, other methods are necessary, which will be described in Sect. 2.3.

Punctuality relates to the deviation from the scheduled arrival and departure times. The headways are of no importance. Unpunctual services are caused by the same conditions that cause irregular services. The key difference between these two concepts can be illustrated by the example that if transit service is systematically two minutes late the punctuality is poor while the regularity is perfect.

\subsection{Impact of service regularity}

Basically, service regularity influences both the supply side and the demand side. For the supply side it influences the capacity efficiency: the distribution of the passengers over the vehicles. A better regularity results in an even distribution of the passengers and thus in lower peaks in occupancy.

For the demand side an improved regularity affects two aspects:

Product appreciation: the service quality for the traveler and his appreciation for it. Current travelers will appreciate transit more because of shorter travel times and less crowded vehicles (see capacity efficiency);

Product attractiveness: A better regularity makes transit more attractive for other travelers, apart from a higher product appreciation also because of shorter waiting times.

Of course the aspects on the demand side are closely related. The first is primarily related to regular travelers, while the second deals with attracting new travelers.

Research shows that a better reliability will attract more transit users (Centrum voor omgevings- en verkeerspsychologie 1998; Bates and Polak 2001; Bruinsma et al. 1999). In these studies reliability is defined as probability that a trip can be 
made according to the expected trip characteristics: travel time, comfort and costs. Improving regularity and punctuality thus play an important role in making transit more attractive.

\subsection{Impact on travel time}

It is a well-known fact that travel time is a key component in all kinds of traveler decisions. Irregular transport services influence in-vehicle times as well as the waiting times, i.e. at the first stop and at transfer nodes. In the case that a transit planner uses average trip times to determine the in-vehicle time, the impact of irregular services on the in-vehicle time is accounted for. The impact on the waiting time, however, is something else. In urban areas travelers tend to arrive randomly at the stop, at least for high frequency services having headways of 12 minutes or less (O'Flaherty and Mangan 1970; Seddon and Day 1974). In case of regular services, average waiting time will be equal to half the headway. A generic formulation for the average waiting time is given by (Osuna and Newell 1972; Heap and Thomas 1976; Welding 1957):

$$
E\left(W_{j}\right)=\frac{E\left(H_{j}\right)}{2} \cdot\left(1+\frac{\operatorname{Var}\left(H_{j}\right)}{E^{2}\left(H_{j}\right)}\right)
$$

where:

$$
\begin{aligned}
E\left(W_{j}\right) & =\text { the expectation of the waiting time at stop } j \\
E\left(H_{j}\right) & =\text { the expectation of the headways at stop } j \\
\operatorname{Var}\left(H_{j}\right) & =\text { the variance of the headways at stop } j
\end{aligned}
$$

Travelers attach a weight of 1.5 up to 2.3 to waiting times in urban transit systems (Van der Waard 1988; Wardman 2001), which makes waiting time an important component of the total trip time.

If instead of the variance of the headways PRDM is used as a regularity indicator, the waiting time for irregular services can be approximated by Hakkesteegt and Muller (1981):

$$
E\left(W_{j}\right) \approx \frac{1}{2} \cdot H_{j} \cdot\left(1+P R D M_{j}^{2}\right)
$$

Note that the definition of PRDM has a large similarity with the coefficient of variation of the headways. Furthermore, the PRDM is only defined for cases having a constant scheduled headway. In situations in which variables headways are expected, for instance two common lines having different frequencies, (2) should be used.

\subsection{Analyzing regularity and transit network design}

In transit network design a trade-off is determined between travelers' benefits and operator's objectives. Given a specific network design an assessment is made of the quality offered to the traveler. In this context different related characteristics can be used: waiting times, frequencies and headways. The traditional approach is to calculate the waiting time using only the frequency (i.e. vehicles per hour, (4)), while in 
reality the regularity plays a role as well (see (2) and (3)). As a result the perceived waiting time is different from the computed waiting time, thus a distinction can be made between perceived frequency and scheduled frequency, or in other words, the perceived headway and the scheduled headway. The perceived headway is defined as the headway that given a regular service would result in the waiting time perceived by the traveler. This implies that the perceived headway is twice the average waiting time given the expected service regularity (equation (5)). The perceived frequency $\left(F_{p}\right)$ can thus be defined as shown in $(6)$. Please note that these formulations are applicable at the level of stops (requiring the index $j$ ) as well as the level of the line.

$$
\begin{aligned}
F & =\frac{60}{H} \\
H_{p} & =2 \cdot E(W) \\
F_{p} & =\frac{60}{H_{p}}=\frac{60}{H\left(1+P R D M^{2}\right)}=\frac{F}{\left(1+P R D M^{2}\right)}
\end{aligned}
$$

where:

$$
\begin{aligned}
F & =\text { Scheduled frequency } \\
F_{p} & =\text { Perceived frequency } \\
H_{p} & =\text { Perceived headway }
\end{aligned}
$$

Figure 1 shows the dependence of the perceived frequency and the irregularity for different scheduled frequencies. It can be seen that if the PRDM is 100\%, i.e. bunching, the perceived frequency is half the scheduled frequency, or in other words, the vehicles operate in pairs.

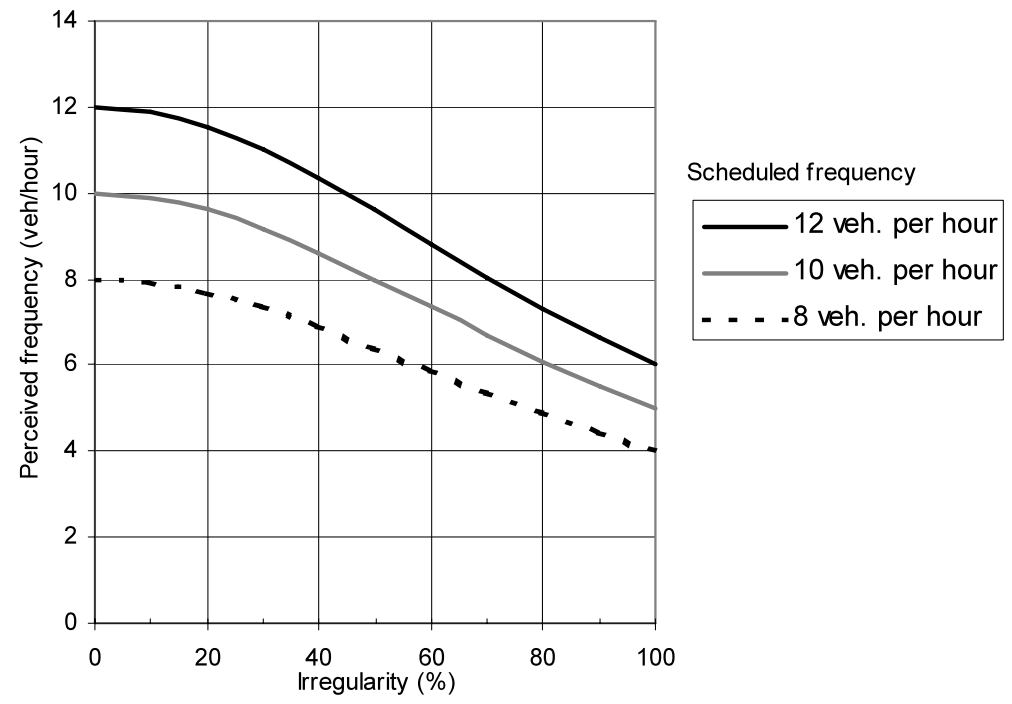

Fig. 1 Relationship between perceived frequency and irregularity 


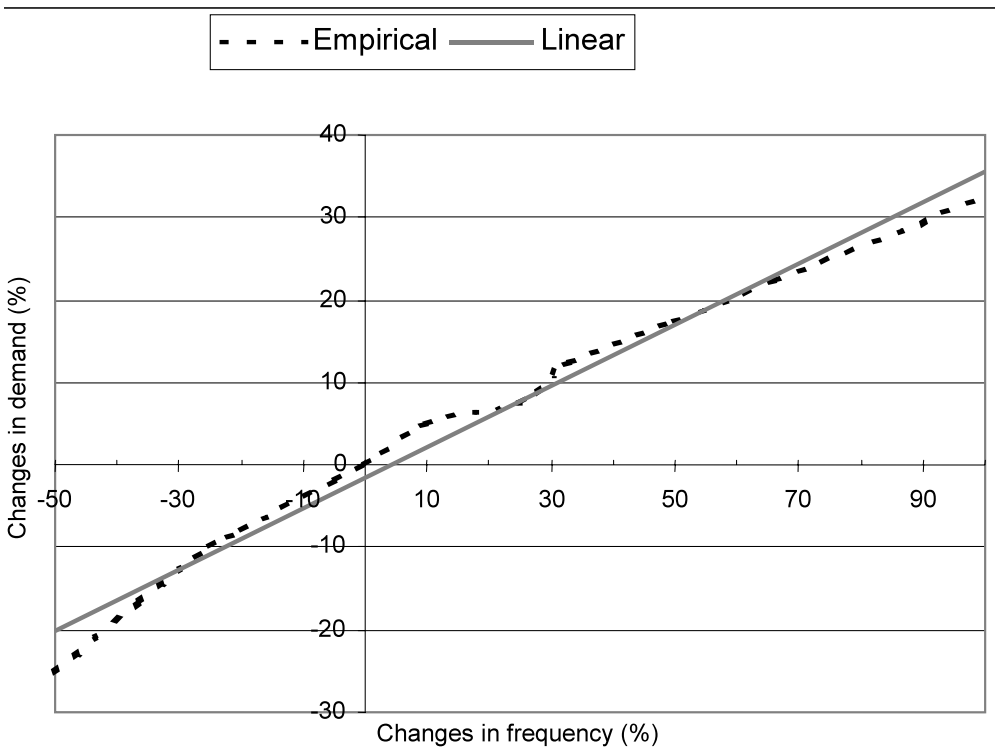

Fig. 2 Relationship between changes in frequency and changes in demand in The Hague (Bureau Goudappel Coffeng 1987)

The perceived frequency can be used to assess the impact of regularity on the level of demand in terms of number of travelers. An empirical study in The Hague (Bureau Goudappel Coffeng 1987) showed the relationship between changes in frequency and in travel demand for urban transit systems (Fig. 2). Since this relationship is found to be nearly linear, elasticity might be used to describe this relationship. If a transit planner intends to propose network structures that require coordination between transit lines, such as two or more lines having the same route, or would like to improve the regularity by splitting lines in two, he should obviously consider the impact of regularity, or irregularity, on the expected performance of the network. As stated before, the traditional approach is to determine the waiting time on the scheduled frequencies only. If the network improvements are primarily focused on improving the regularity, transit planners might use a qualitative assessment in terms of a substantial improvement of regularity. However, if it would be possible to determine the perceived frequency, transit planners could easily determine the full impact of the proposed network changes on the performance of the transit system, including the impact on the service regularity. The next section presents such a tool.

\section{Regularity analysis tool}

The purpose of the tool is to make an estimate of the regularity for a given stop, given a proposal for a network configuration, especially in the case of parallel lines having the same route. Given that estimate, the impact on the level of demand can be determined using the concept of the perceived frequency. The transit planner can then decide whether the expected revenues are in balance with the additional costs related to the proposed change in network configuration (see Fig. 3). 
Fig. 3 Workflow using the regularity analysis tool to assess the impact of network changes

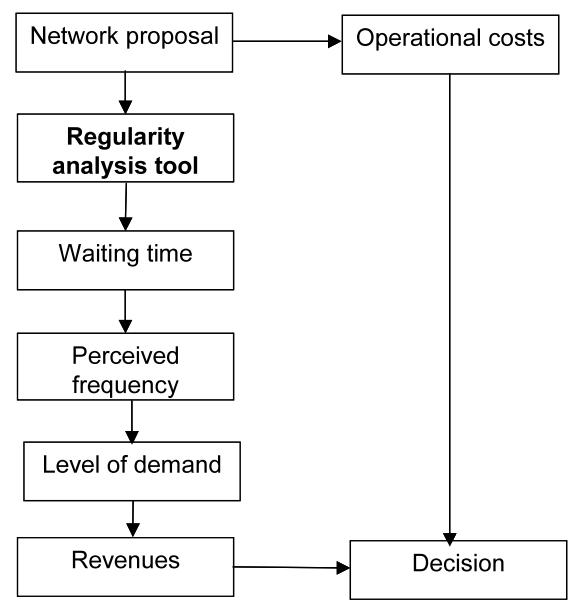

The input of the tool is a description of the proposed network configuration such as the number of lines, their frequencies, and whether it is intended to apply a coordinated timetable or not. Furthermore, a description of the punctuality of the transport services is required in terms of a probability distribution of the vehicle arrivals or departures. Ideally this description should be based on actual data of the system performance today, such as collected with an operations monitoring system such as the TRITAPT-system (Bureau Goudappel Coffeng 1987). Otherwise street research is necessary or empirical data of comparable lines can be used.

The estimation procedure works as follows:

Timetable generation: Given the set of lines, their frequencies and the coordination strategy a timetable for the arrival and departure of the vehicles at each relevant stop is generated;

Simulation of operational performance: Given the probability distribution for each line, a randomization procedure determines a series of the actual arrival and departure times for each vehicle and each stop. This procedure is repeated for a given number of iterations. The arrival and departure times are sorted and stored;

Calculation of the regularity: Given the simulated arrival and departure times at the stops the PRDM is calculated (equation (1)). In case of unequal headways, this step determines the average headway and its variance.

Determination of the waiting time and the perceived frequency at the stops: Given the headway characteristics or PRDM for each stop the expected waiting time is determined (equations (2) and (3), respectively), which then is used to calculate the perceived headway (twice the expected waiting time) and thus the perceived frequency.

Estimation of the impact on demand: given the calculated change in perceived frequency an estimate can be made of the relative change in patronage, for instance using elasticity as suggested in Sect. 2.4.

This procedure can be repeated for all periods of the day. The sum of the changes in demand per period determines the expected revenues, which in general should outweigh the costs involved. 


\section{Case study: Koninginnegracht in The Hague}

This section presents an application of the tool for the route called Koninginnegrachtroute in the city of The Hague in The Netherlands (Fig. 4). This route starts at The Hague Central Station and ends in Scheveningen, a sea-side resort. It is served by

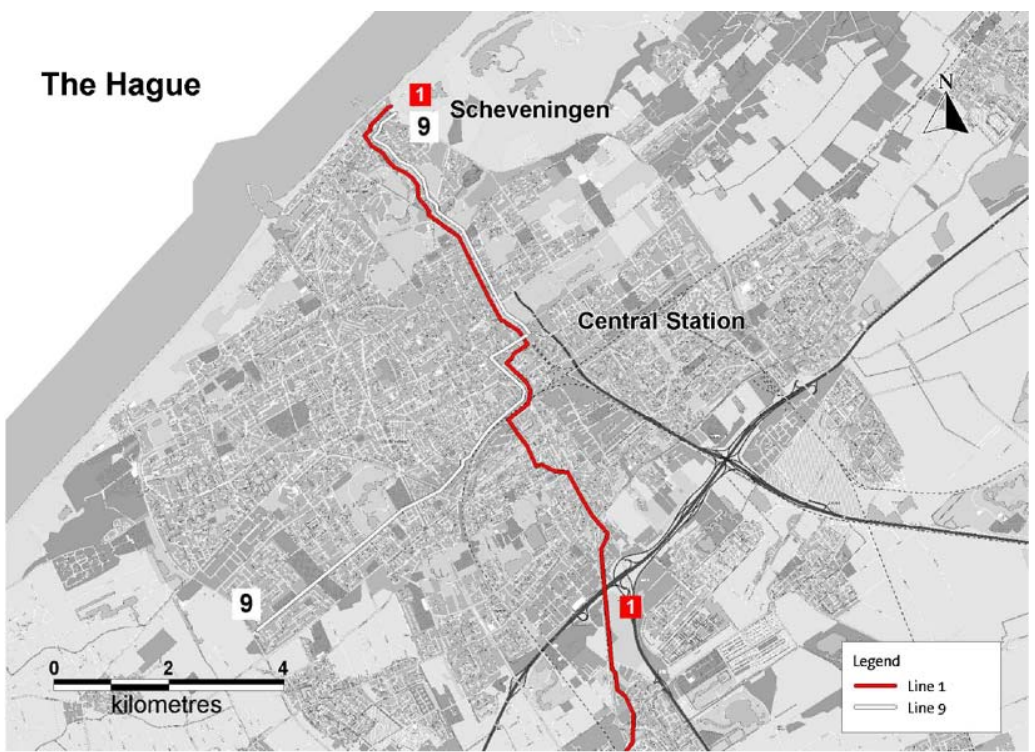

A

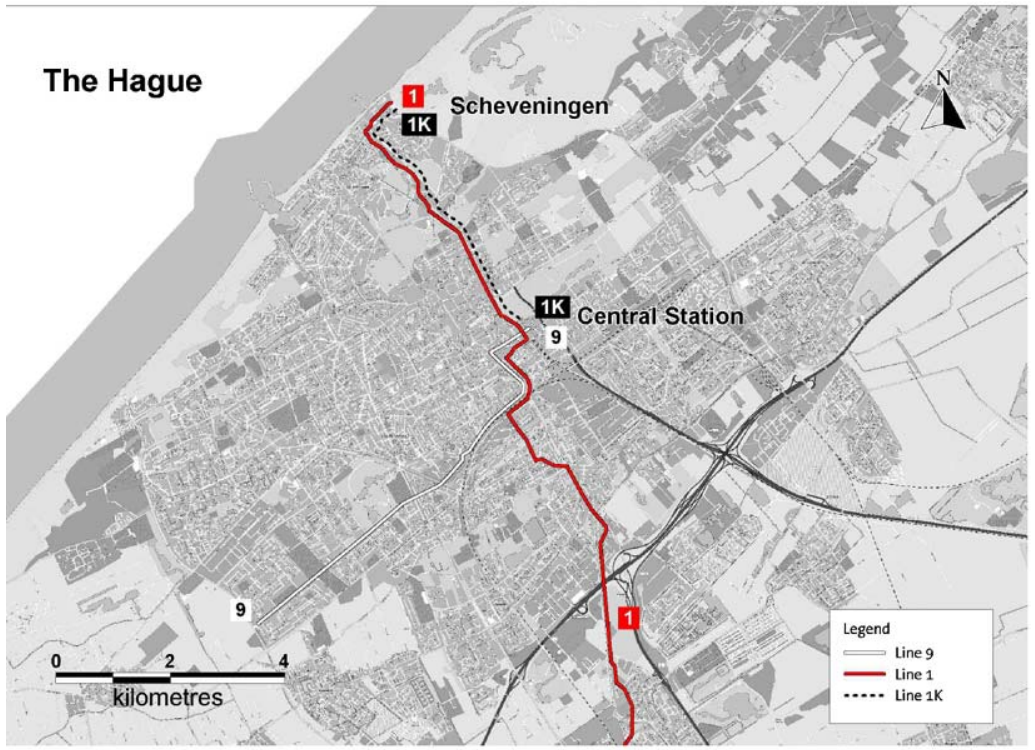

B

Fig. 4 Network layout for the case study 
two tramlines, 1 and 9, which have different starting points. Tramline 1 starts in the city of Delft, while tramline 9 starts in the southern part of The Hague. The regularity of these two tramlines on this route is considered to be poor, because of the long routes these lines have before joining on their common route part. Therefore, it is proposed to limit the service on the Koninginnegracht-route to tramline 1 only, and to introduce an additional service (tramline $1 \mathrm{~K}$ ) between The Hague Central Station and Scheveningen (Fig. 4). Tramline 9 is reduced to the route between the southern part of The Hague and The Hague Central Station.

The consequences of this change in network structure are diverse. Splitting tramline 9 into a reduced tramline 9 and tramline $1 \mathrm{~K}$ requires more vehicles. Furthermore, travelers from The Hague South to destinations close to the Koninginnegracht-route have to make a transfer, although this effect might be limited because of the existence of an alternative tramline connecting The Hague South and Scheveningen (tramline 8). The key benefit of the network change is the improved service quality on the Koninginnegracht-route. A qualitative assessment of this improvement is that the current situation shows a very poor regularity, while in the new situation coordinated and regular transit services are possible. An optimistic estimate might be that the quality changes from highly irregular to highly regular transit services, implying a doubling of the perceived frequency, which would lead to an increase of demand of about $35 \%$ (using Fig. 2). Given the importance of a proper assessment of the benefits for the regularity on this route, a more sophisticated approach is required and thus the tool described in Sect. 3 was used for a quantitative assessment.

The analysis deals with both directions on this route. For each direction an assessment is made for the regularity effects at the start of the common route. The data with respect to the current probability distributions at these stops is collected with the TRITAPT-monitoring system (TU Delft 1997-2002). For the new tramline 1K punctual services are assumed. Using empirical data for The Hague (Bureau Goudappel Coffeng 1987), the elasticity for the relationship between changes in frequency and travel demand is estimated to have a value of 0.36 (R-square of 0.99 ), which states that a change of the (perceived) frequency of $1 \%$ will result in a change in demand of $0.36 \%$.

Tables 1 and 2 show the results for both directions for the two peak periods (for a complete analysis see van Oort 2003). These tables show the current supply characteristics, i.e. frequencies and coordination strategy, and the resulting performance characteristics: waiting times, perceived frequencies, as well as change in perceived frequency and the related change in demand.

It can be seen that in the direction of Scheveningen (Table 1) the regularity will still remain rather poor: the PRDM is estimated to be $46 \%$. As a result the change in perceived frequency and the related change in demand remain small. This is due to the relative high irregularity of tramline 1 when it arrives at The Hague Central Station. The proposed changes in the network have no influence on the regularity of the route between Delft and the starting point of the Koninginnegracht-route, and should therefore be considered as a given fact. This predefined irregularity nearly precludes improving the regularity by replacing tramline 9 by tramline $1 \mathrm{~K}$.

In the other direction (Table 2), however, the resulting regularity is rather good: a PRDM of $20 \%$. As a result the changes in perceived frequency and level of demand 
Table 1 Impact on regularity and level of demand for the Koninginnegracht route from The Hague Central Station to Scheveningen

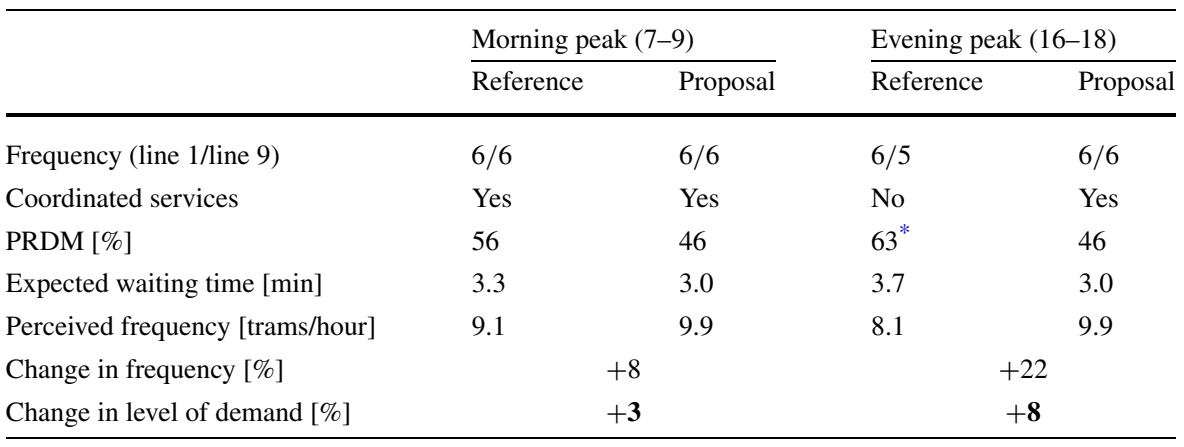

*Estimated using the expected headway and its variance

Table 2 Impact on regularity and level of demand for the Koninginnegracht route from Scheveningen to The Hague Central Station

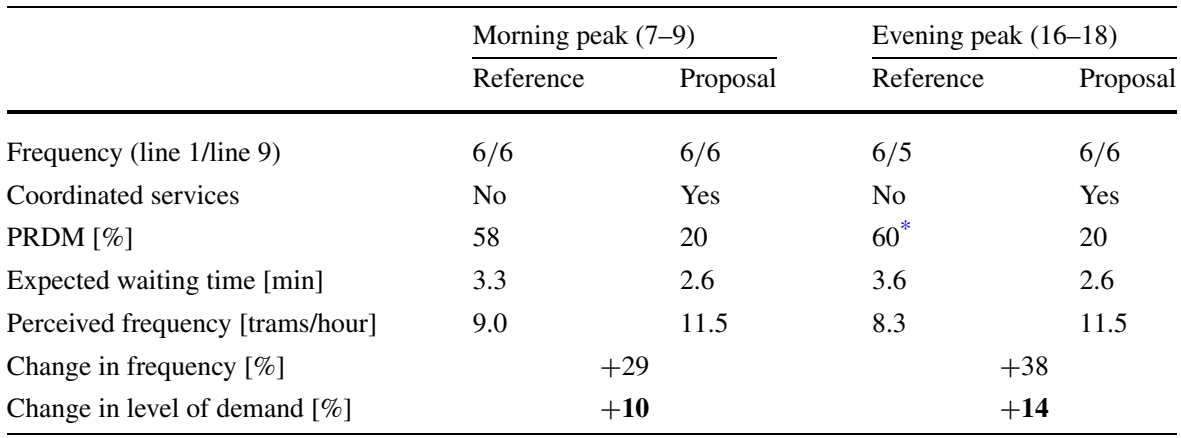

*Estimated using the expected headway and its variance

are substantially higher: up to $38 \%$ and $14 \%$, respectively. The largest differences are found in the case that coordinated services are introduced.

The differences between the two directions show two interesting phenomena. First, if one of the lines is already irregular at the beginning of the route it is not possible to achieve a substantial improvement in regularity by introducing a shortturn line. In this case other measures for improving the regularity of tramline 1 are more appropriate. Second, the findings for the direction to The Hague Central Station show that the reference situation is less bad than it was assumed. Therefore, the impact of the improved regularity is smaller than it might be expected based on an intuitive approach.

The net change in demand as estimated with the analysis tool is $9 \%$, which is more than three times lower than the rough estimate of 30\%. This analysis thus clearly shows that a quantitative analysis is essential for judging such network proposals. 


\section{Quick scan}

When designing urban transit networks it is interesting to have an indication whether combining (parts of) two lines on the same route provides real benefits for the travelers, instead of having a detailed analysis such as given in the previous section. This is relevant if parallel line structures or short-turn services are considered, or when it is intended to introduce a dedicated transit lane, which is used by a number of transit lines.

Therefore, the tool has been used to develop graphs that can be adopted as a quick scan to indicate the potential change in regularity if two lines operate on a single route with or without coordination. These graphs are based on the standard deviation of the punctuality of each line. The analysis has been performed for the case of two lines having a frequency of 6 vehicles per hour. In the coordinated case these lines are scheduled in such a way that the average headway is 5 minutes. If there is no coordination the second line departs 1 minute later than the first.

Figure 5 shows the regularity level as a function of the standard deviation of the punctuality for both lines in the uncoordinated case. The horizontal axis shows the standard deviation of the punctuality of the first line, while each curve relates to a punctuality standard deviation of the other line. The vertical axis represents the value of the regularity defined by the PRDM. If both lines are very punctual, the regularity for the combination of both lines is very poor (80\%). In fact, an increase of the variation of the punctuality leads to a better regularity, the best value in the graph being $52 \%$.

Comparing this graph with the coordinated case (Fig. 6) clearly shows that coordinated services have a much better performance. In the case of very punctual services the regularity is perfect $(0 \%)$. However, in the case of unpunctual services the regularity deteriorates to $55 \%$.

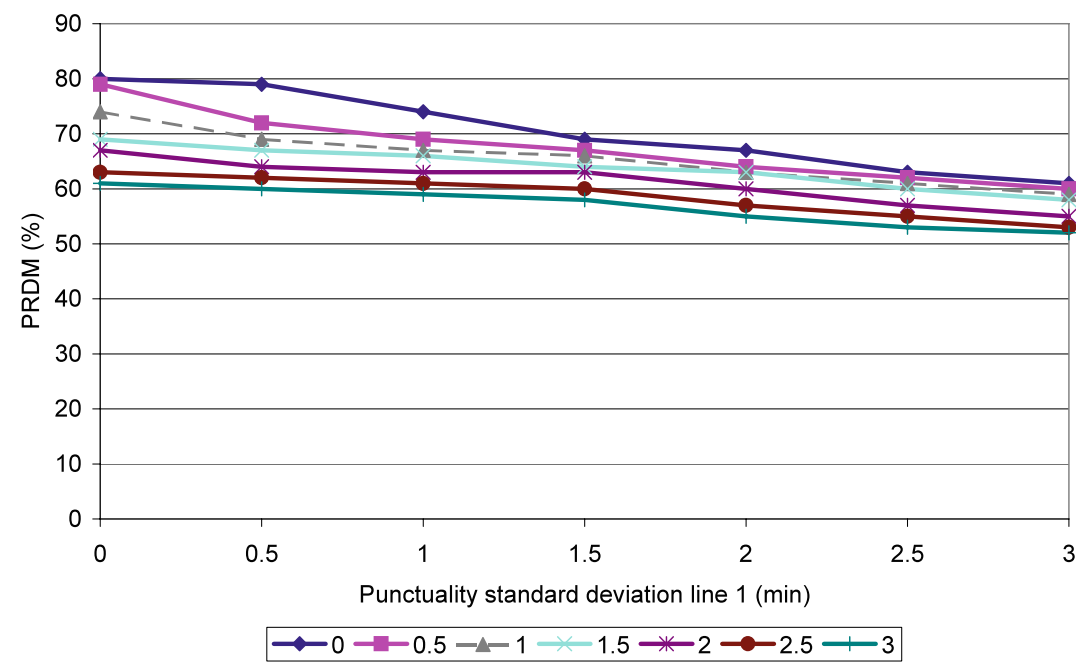

Fig. 5 Regularity (PRDM) as a function of the punctuality of line 1 for different values of the punctuality of line 2, uncoordinated case 


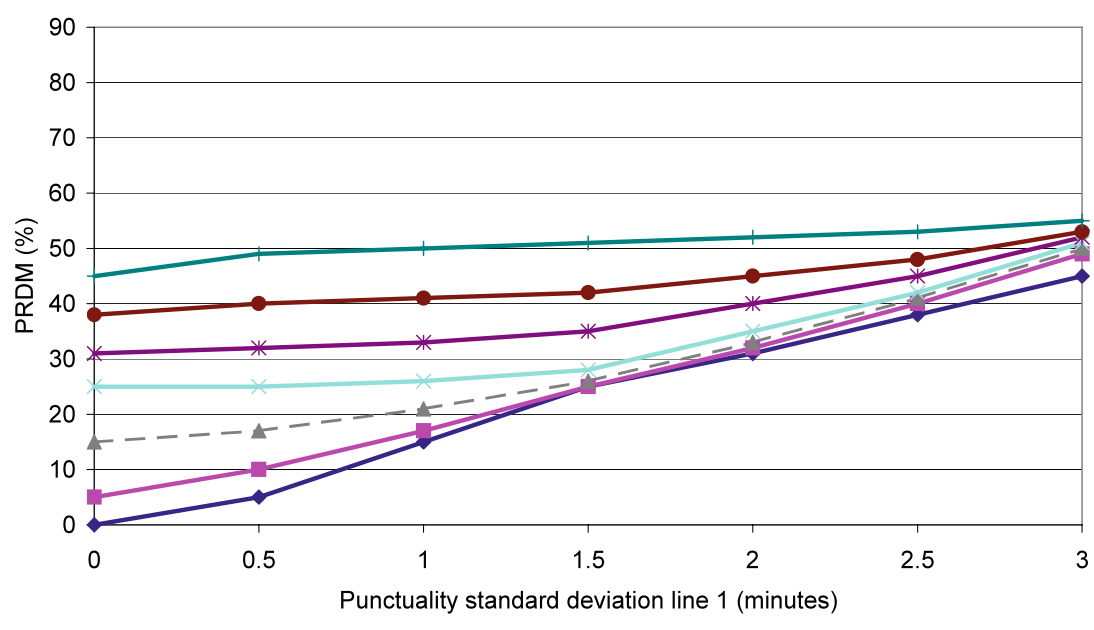

$\rightarrow 0-0.5-\Lambda-1 \div 1.5 \div-2 \rightarrow 2.5 \div-3$

Fig. 6 Regularity (PRDM) as a function of the punctuality of line 1 for different values of the punctuality of line 2, coordinated case

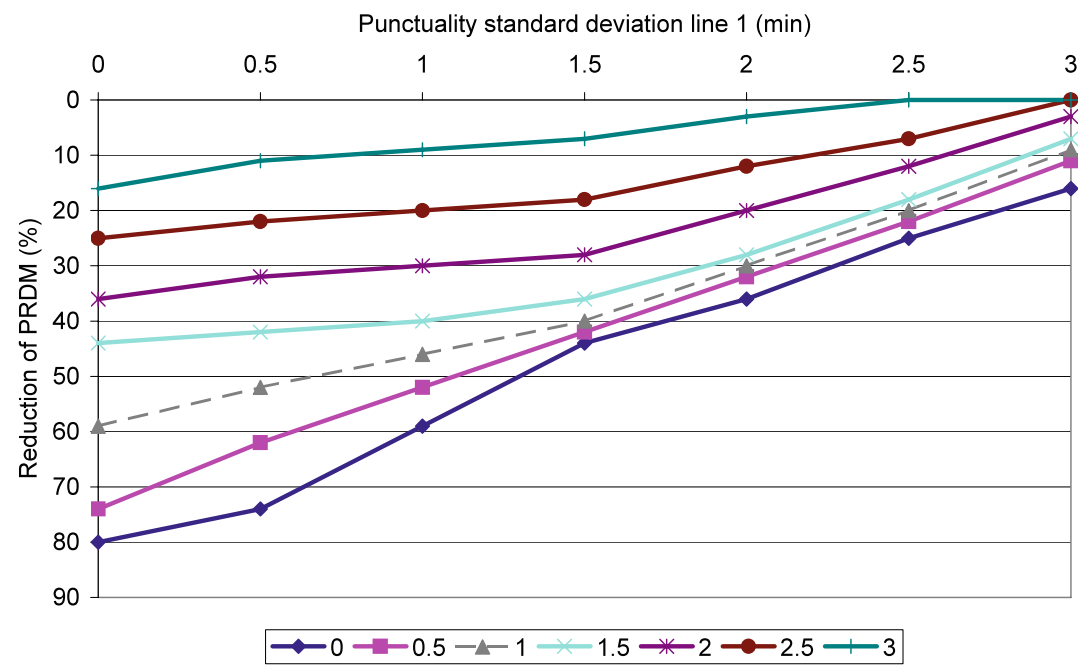

Fig. 7 Reduction of the PRDM as a function of the punctuality of line 1 for different values of the punctuality of line 2 due to coordinating the transport services

These graphs can be used to assess the impact of coordinating services or of reducing the variation in the punctuality. Figure 7 shows the reduction of the PRDM as a result of coordinating services. For highly unpunctual services the impact on the regularity is limited. For services having a medium punctuality (standard deviation of 1.5 minutes) the PRDM can be reduced with $40 \%$, while in the case of very punctual services a reduction of $80 \%$ is feasible. 


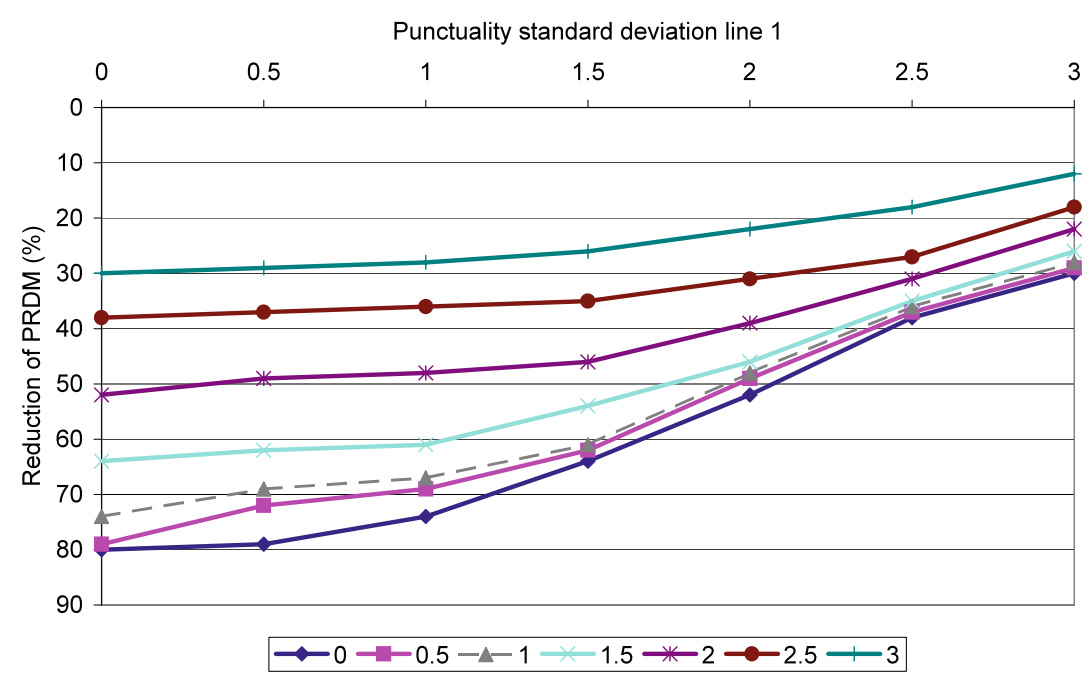

Fig. 8 Reduction of the PRDM as a function of the punctuality of line 1 for different values of the punctuality of line 2 due to coordinating the transport services and improving the punctuality by reducing the standard deviation by 1 minute for both lines

The results for the situation in which coordinating of services is combined with improving the punctuality are shown in Fig. 8. This clearly shows that in the case of unpunctual services a substantial improvement is possible. The largest improvement, however, is found in the case of lines having a medium punctuality level. The reduction of the PRDM then varies between 17 and $20 \%$. Of course, in the case of punctual services the benefits of improving the punctuality are small.

These graphs can be applied to the case of the tramlines described in the previous section. In the reference case there are two lines with poor punctuality: the standard deviation of both lines is about 3 minutes (direction Scheveningen). In the new situation the punctuality of tramline 1 remains unchanged, while the punctuality of tramline $1 \mathrm{~K}$ is very high. Using Fig. 6 it can be seen that the original value for the PRDM was $55 \%$ and in that in the new situation it will be $45 \%$. If the punctuality of tramline 1 is improved to, say, a standard deviation of 1.5 minutes the PRDM will be reduced to $25 \%$. Furthermore, it can be seen that for the regularity it is not necessary to have a perfect punctuality for the short-turn tramline $1 \mathrm{~K}$. If both lines have a standard deviation of 1.5 minutes the PRDM becomes only $3 \%$ higher: $28 \%$. In this case improving the punctuality of tramline 1 is more beneficial than introducing a short-turn service.

\section{Conclusions}

This paper described a tool that can be used to assess the impact of network changes on the regularity on a transit route and on the level of transit demand. Typical issues that can be analyzed are combining lines or parts of lines on a route or introducing short-turn services. The tool can use actual data on the punctuality of the transit 
system. The application of such a tool was illustrated in two ways. The case study showed that the use of such a tool leads to more realistic estimates than the traditional approach using a quantitative analysis: the realistic estimate appeared to be a factor 3 lower. Second, a set of graphs was developed which can be used for a quick scan when considering network changes. These graphs can be used to assess the effect of coordinating the schedules and of improving the punctuality.

The tool focuses on the impact on regularity and the level of demand. Changes in the regularity also influence the capacity efficiency, which might influence the operational costs. Therefore, the tool might be extended in the future to incorporate operational costs.

Open Access This article is distributed under the terms of the Creative Commons Attribution Noncommercial License which permits any noncommercial use, distribution, and reproduction in any medium, provided the original author(s) and source are credited.

\section{References}

Barnett A (1974) On controlling randomness in transit operations. Transp Sci 8(2):102-116

Bates J, Polak J (2001) The valuation of reliability for personal travel. Transp Res E 37:191-229

Bruinsma FR, Peeters P, Rietveld P, van Vuuren DJ (1999) Reliability of public transport chains. Tijdschr Vervoer 35:93-111 (in Dutch)

Bureau Goudappel Coffeng (1987) Modal split study in the city of The Hague (in Dutch)

Carey M (1998) Optimizing scheduled times, allowing for behavioural response. Transp Res B 32(5):329_ 342

Centrum voor omgevings- en verkeerspsychologie (1998) Reliability of means of transport, Groningen (in Dutch)

Chowdhury S, Chien S (2001) Dynamic vehicle dispatching at intermodal transfer station. In: Transportation Research Board 80th annual meeting, Washington, DC

Fu L, Liu Q (2003) A real time optimization for dynamic scheduling of transit operations. In: Transportation Research Board 82th annual meeting, Washington, DC

Hakkesteegt P, Muller ThHJ (1981) Research increasing regularity. Verkeerskundige werkdagen, pp 415436 (in Dutch)

Heap RC, Thomas TH (1976) The modelling of platooning tendencies in public transport. Traffic Eng Control 8(9):360-362

Israeli Y, Ceder A (1996) Public transportation assignment with passenger strategies for overlapping route choice. In: Lesort JB (ed) Transportation and traffic theory. Elsevier Science, Amsterdam

Muller ThHJ, Furth PG (2000a) Integrating bus service planning with analysis, operational control and performance monitoring. In: ITS 10th conference proceedings, Washington, DC

Muller ThHJ, Furth PG (2000b) Conditional bus priority at signalized intersections: better service with less traffic disruption. Transp Res Rec 1731:23-30. Transportation Research Board, Washington, DC

O'Flaherty CA, Mangan DO (1970) Bus passengers waiting time in central areas. Traffic Eng Control 11:419-421

Osuna EE, Newell GF (1972) Control strategies for an idealized public transport system. Transp Sci 6(1):52-72

Seddon PA, Day MP (1974) Bus passengers waiting times in greater Manchester. Traffic Eng Control $15: 422-445$

TU Delft (1997-2002) TRIp time analysis in public transport (TRITAPT). Computer application, Delft

Van der Waard J (1988) The relative importance of public transport trip time attributes in route choice. PTRC, London

van Oort N (2003) Impact of the timetable and performance of public transport on network design. TU Delft/HTM The Hague (MSc-Thesis) (in Dutch)

Wardman M (2001) Public transport values of time. Working paper 564, Institute of Transport Studies, University of Leeds, Leeds

Welding PI (1957) The instability of a close interval service. Oper Res Q 8(3):133-148 